\title{
Frontal cortical lesions and prestimulus inhibition of the rat's acoustic startle reaction*
}

\author{
GEOFFREY R. HAMMOND \\ University of Western Australia, Nedlands, Western Australia 6009, Australia
}

\begin{abstract}
This paper reports two experiments dealing with the effects of lesions of the frontal cortex on the amplitude of the rat's acoustic startle reaction, both under control (unstimulated) conditions and under conditions where the response is inhibited by delivery of a visual or auditory stimulus just prior to response elicitation. While in no case was brain damage observed to lead to any derangement of the prestimulus inhibition of the response, some animals showed exaggerated levels of reactivity on control trials postoperatively. Correlations between location of brain damage and postoperative responsivity indicated that damage to the medial sector of the frontal cortex was critical in obtaining augmented levels of startle.
\end{abstract}

Inhibitory control of the amplitude of the acoustic startle reaction by antecedent stimuli which need not themselves have detectable response consequences has been described extensively (Hoffman \& Searle, 1965; Ison \& Hammond, 1971). Briefly, both visual and auditory prestimuli provide inhibition, the degree of inhibition obtained being a reliable function of the interstimulus interval (ISI) separating the antecedent and response-eliciting stimuli: the degree of inhibition initially increases with increasing ISI to some maxima (around $40 \mathrm{msec}$ in the case of an auditory prestimulus, $60 \mathrm{msec}$ in the case of a visual prestimulus), thereafter decreasing gradually to control response levels obtained by presentation of the primary response-eliciting stimulus alone.

Potential accounts of this form of response inhibition in terms of either a sensory dampening framework, where the prestimulus is considered to degrade the sensory impression of the response-eliciting stimulus, or in terms of muscular refractoriness, where the response is assumed to be elicited in a state of relative refractoriness initiated by the prestimulus, may be discounted. Firstly, it has been shown that the suppression of the second response obtained when two startle-eliciting stimuli are presented sequentially, which is dependent on the intensity of the first member of the stimulus pair, is not lessened by the appearance of a prestimulus just prior to the first member of that stimulus pair (Ison \& Krauter, 1973). Furthermore, modulation of acoustic input by known sensory control mechanisms shows a number of inconsistencies with the

\footnotetext{
*The research reported here was carried out while the author was a PhD candidate at the University of Rochester, and formed part of a thesis submitted in partial fulfillment of the requirements of that degree. The author expresses his appreciation to J. R. Ison, G. J. Thom as, and D. W. McAdam for their advice throughout this research, and to Jean Conlon for her training in histological methods. Partial support for the research was provided from NSF Grant GB-14814, NIMH Grant MH-13581, and NINDS Grant NS0-8456.
}

behavioral data with respect to both amount and temporal pattern of inhibition. Secondly, electromyographic recordings taken during a typical prestimulus-startle stimulus sequence have shown that a prestimulus need not initiate activity in a particular muscle group in order for it to exert effective inhibition on the subsequently elicited activity of that group (Hammond, McAdam, \& Ison, 1972). For these reasons, we have argued that prestimulus inhibition is most probably the expression of a central behavioral inhibitory system which is sensitive to momentary changes in external stimulation. In particular, attention is drawn to a series of experiments by Clemente and his associates (see Clemente, 1968, for a review) which has shown that punctate electrical stimulation of the orbital gyrus of the frontal cortex in cats initiates a cycle of inhibition such that subsequently elicited responses are depressed in amplitude. The similarity of the effects noted in the cat preparation to the behavioral functions obtained in the intact rat, together with the variety of response systems which have been shown to be subject to inhibitory modulation from the frontal cortex, led to the suggestion that frontal cortex in the rat may form part of a similar behavioral inhibitory system, and that prestimulus inhibition might be effected through such a system. Some evidence does exist to implicate the frontal cortex in the rat in behavioral suppression. Thus, surgical damage to frontal cortical areas has been reported to lead to hyperactivity (Richter \& Hawkes, 1949, cited in Brutkowski, 1965; Lynch, 1970), which may be interpreted as a release phenomenon in terms of a lesion-induced impairment in behavioral inhibition; response habituation has been found to be retarded markedly following frontal cortical ablation (Griffin \& Pearson, 1968; Roydes, 1970), although, in balance, damage to a variety of brain sites has been found to have a similar effect (Thompson \& Spencer, 1966); and finally, rats with frontal cortical damage have been 
found to be notably deficient in suppressing an acquired response to a previously rewarded, but currently nonrewarded, stimulus, a set of findings generally interpreted as a consequence of lesion-induced dysfunction of "internal inhibition" (see Brutkowski, 1965 , for a general review).

These lines of evidence led us to examine the effects of frontal cortical ablations on measures of startle responsivity where the startle-eliciting stimulus was presented alone (control condition) and where the response was inhibited by an antecedent visual or auditory stimulus.

\section{GENERAL METHOD}

\section{Animals}

Experimentally naive male albino rats, purchased from the Holtzman Company, were used. They were housed individually and kept on a continuous food and water supply except for the 18-24 h preceding surgery.

\section{Apparatus}

The animals were confined during testing in a small perforated Plexiglas chamber ( $8 \times 2.5 \times 3.5$ in. high), which was held firmly between compression springs within an aluminum superstructure. The startle stimulus was a $9,000-\mathrm{Hz}$ tone generated by an oscillator, which, following timing and amplification, was delivered via a high-frequency tweeter directed at the experimental cage. The tone was $20 \mathrm{msec}$ in duration, with a rise and decay time of $5 \mathrm{msec}$. Two other speakers directed at the cage provided a white noise background and a white noise prestimulus. Two light bulbs mounted on the cage provided a visual prestimulus. Magnitude of the startle reaction was detected by an accelerometer mounted on the cage and recorded on a polygraph as millimeters of pen deflection, a measure which is linearly related to the acceleration imparted to the cage by the animal's reaction. The onset of a trial was controlled by a tape timer programmed to initiate trials with an average interval of $45 \mathrm{sec}$ (range, 30-60 sec). The apparatus was housed in a sound-attenuating testing room. A more detailed description is available in Ison and Hammond (1971).

\section{Surgical Procedures}

Following pretreatment with atropine sulfate, surgery was carried out under deep sodium pentobarbital anesthesia. With the animal secured in a stereotaxic head holder, the skull was exposed and small bone flaps removed bilaterally from over the frontal region. The dura was then cut, and for the experimental animals, tissue aspirated with gentle suction through a blunted 18-G hypodermic needle under direct visual control. For control animals, the dura was left intact and no intentional brain damage was produced. The skull openings were then covered with Gelfoam moistened with isotonic saline, and the scalp wound closed with interrupted silk sutures. Postoperatively, the animals were given broad-spectrum antibiotic treatment intramuscularly.

\section{Histological Procedures}

At the conclusion of behavioral testing, the animals were sacrificed with an overdose of sodium pentobarbital and perfused intracardially with physiological saline followed by $10 \%$ Formalin in saline. Following further hardening in Formalin, the brains were either embedded in celloidin and cut serially at a thickness of 48 micra or cut with frozen tissue technique at the same thickness. When cut frozen, all brains were placed in $30 \%$ ethyl alcohol overnight prior to cutting to reduce ice crystal formation during freezing. Every third section through the damaged area was saved and stained with cresyl violet.

\section{EXPERIMENT I}

A total of 11 animals were given two behavioral test series, one preoperative and a second following either extensive bilateral damage to frontal cortex (experimentals, $\mathrm{N}=6$ ) or a similar surgical experience in which no deliberate brain damage was done (controls, $\mathrm{N}=5$ ).

\section{Behavioral Procedures}

Each behavioral test series consisted of 10 control trials, where the startle pulse was presented alone, 15 trials where the startle pulse was preceded by an auditory prestimulus at one of three ISIs, and 15 trials where the startle pulse was preceded by a visual prestimulus at the same ISIs. The ISIs (from the onset of the prestimulus to the onset of the startle pulse) were measured at 40,60 , and $180 \mathrm{msec}$. The presentation order of each of these eight conditions (the control trials were arbitrarily designated as "visual" or "auditory" for the sake of data analyses and presentation) was determined from a table of random numbers for each animal. A 3-min settling-down period in the testing chamber was allowed before the test series was begun.

The auditory prestimulus (duration $=20 \mathrm{msec}$ ) was set at an intensity of $92-94 \mathrm{~dB}$ in a continuous background white noise level of $69-71 \mathrm{~dB}$; the intensity of the visual prestimulus (duration $=20 \mathrm{msec}$ ) was $40 \mathrm{fc}$ in a continuous background illumination of $.06 \mathrm{fc}$. The startle pulse was set at a duration of $20 \mathrm{msec}$ and an intensity of $118 \mathrm{~dB}$. All acoustic intensities are given in $\mathrm{dB}$ re .0002 dynes $/ \mathrm{cm}^{2}$.

All animals (weight range from 340 to $410 \mathrm{~g}$ at surgery) received their postoperative test series after a recovery period ranging from 3 to 14 days following surgery. Preoperative and postoperative tests were given at the same time of day to minimize cyclical fluctuations in responsivity.

\section{Results and Discussion}

Behavioral results from the operated controls are given in Fig. 1, which shows preoperative and postoperative response amplitudes under each condition for each S. ${ }^{1}$ It is clear that all Ss show very similar temporal patterns of response inhibition to both the auditory and visual prestimuli; these response profiles coincide with those noted previously in untreated animals run under similar conditions (Ison \& Hammond, 1971). Furthermore, these response profiles remained stable across tests, as did the control response level for most of the animals; one S (S 4) displayed a consistently lower control response level in the second test $(t=2.24$, $\mathrm{df}=18, \mathrm{p}<.05)$.

Data from the brain-damaged animals are depicted in Fig. 2. As with the control animals, no systematic changes in the pattern of response under the various prestimulus conditions were noted. In all cases, clear inhibition of response under the preoperatively effective conditions was obtained in the postsurgical test. This failure to note any modification of stimulus inhibition postoperatively argues against the attribution of any essential involvement of the ablated regions in the mediation of prestimulus inhibition of the startle reaction.

Although there were no noticeable postoperative changes in the pattern of prestimulus inhibition, five out of the six experimental rats tested showed a marked elevation of control response levels postoperatively. 
These five experimental animals showed postoperative control response amplitudes ranging from $38 \%$ to $103 \%$ of the preoperative response level, with a median increase of $77 \%$. In all of these cases, statistical reliability was met (ps $<.05$ or less, as shown by $t$ values taken from analyses of variance conducted for each $\mathrm{S}$ ). The potentiation of response amplitude noted in most of these Ss is in contrast to the control data, and is suggestive of a lesion-induced release of response inhibition. Thus, while these data do not provide any support for the contention that stimulus-produced inhibition of startle amplitude involves the ablated frontal cortical regions, the observed postoperative elevation of control response amplitude is consistent with the implication of frontal cortical areas of the rat in some sort of general behavioral inhibition.

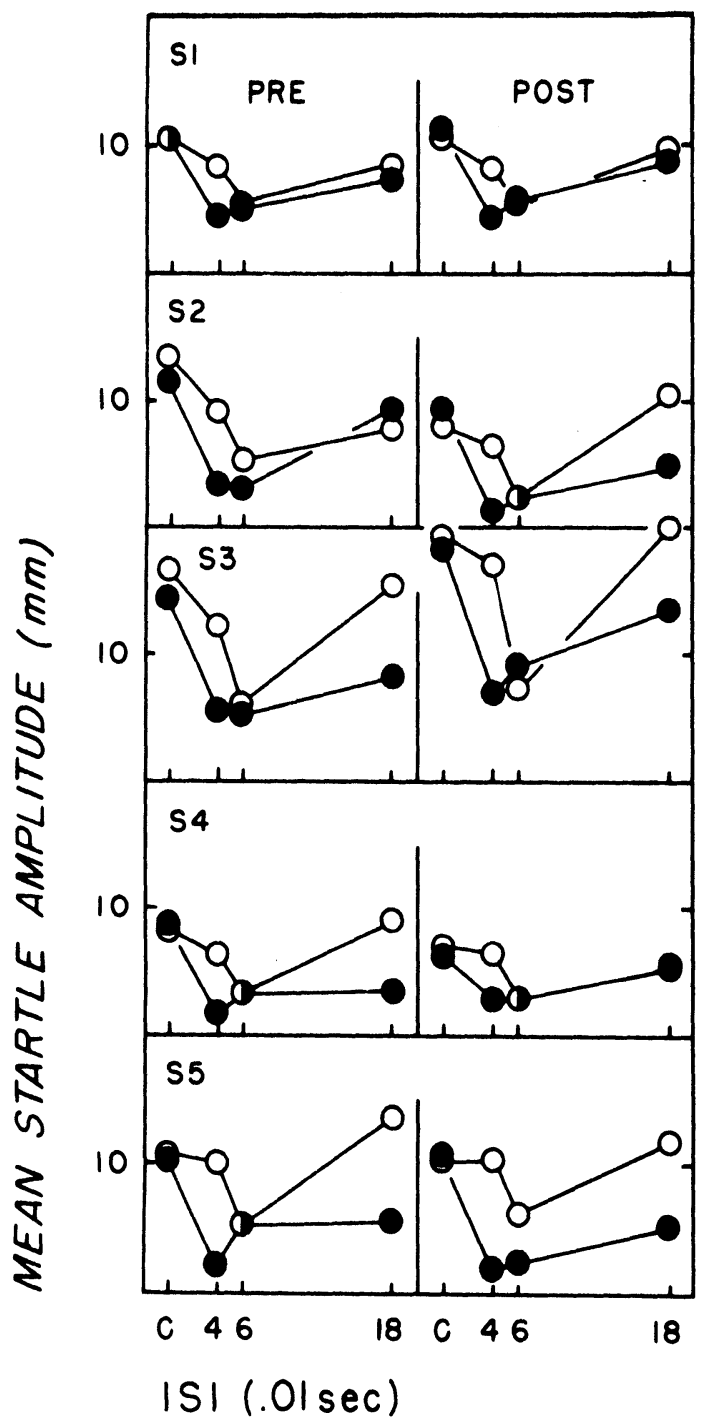

Fig. 1. Mean preoperative (PRE) and postoperative (POST) response amplitudes for the operated control animals under control conditions (C) and under conditions where the startle pulse was preceded by the auditory prestimulus (filled circles) and the visual prestimulus (open circles) at the ISIs shown.

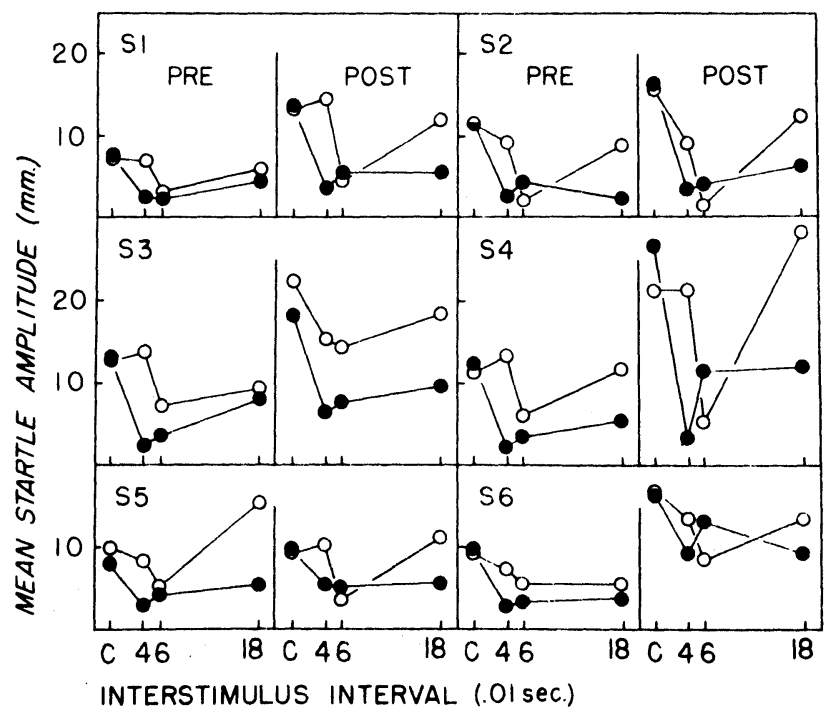

Fig. 2. Mean preoperative (PRE) and postoperative (POST) response amplitudes for animals with frontal cortical ablations under control conditions (C) and under conditions where the startle pulse was preceded by the auditory prestimulus (filled circles) and the visual prestimulus (open circles) at the ISIs shown.
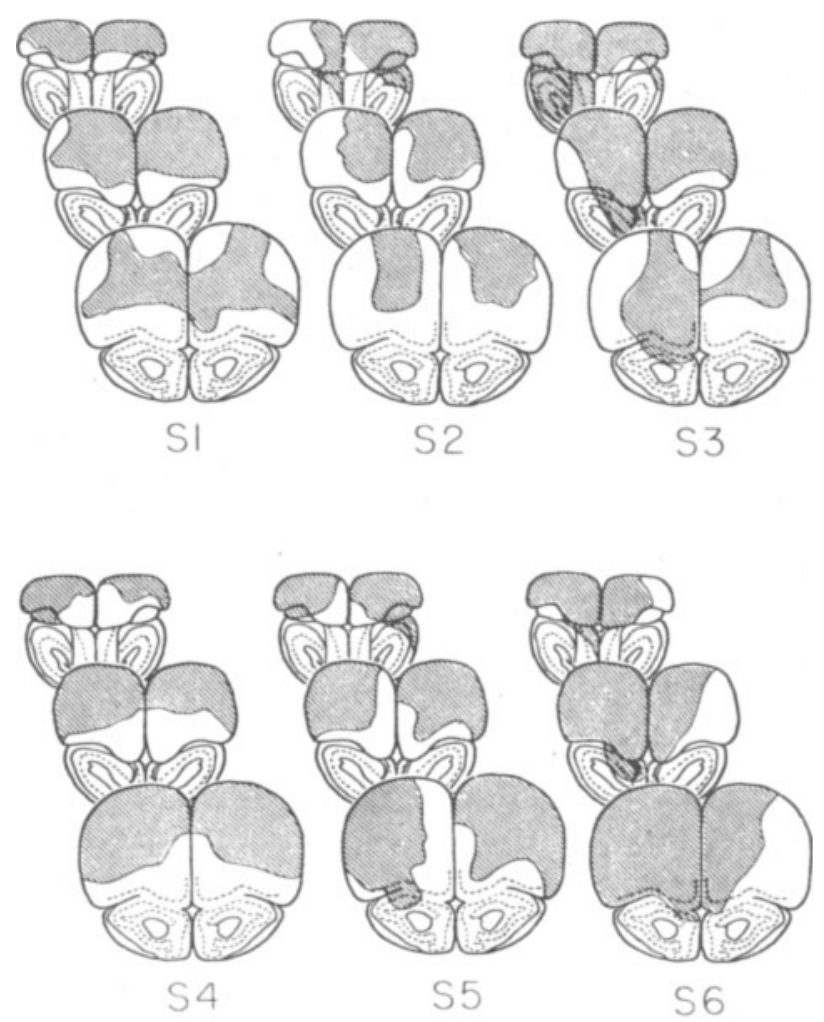

Fig. 3. Reconstructions of brain damage (hatched area) for each animal on illustrations of three frontal sections after König and Klippel (1963).

Histological. Illustrations of ablations on three frontal planes for each experimental S are given in Fig. 3. As can be seen, all sustained substantial damage to frontal 


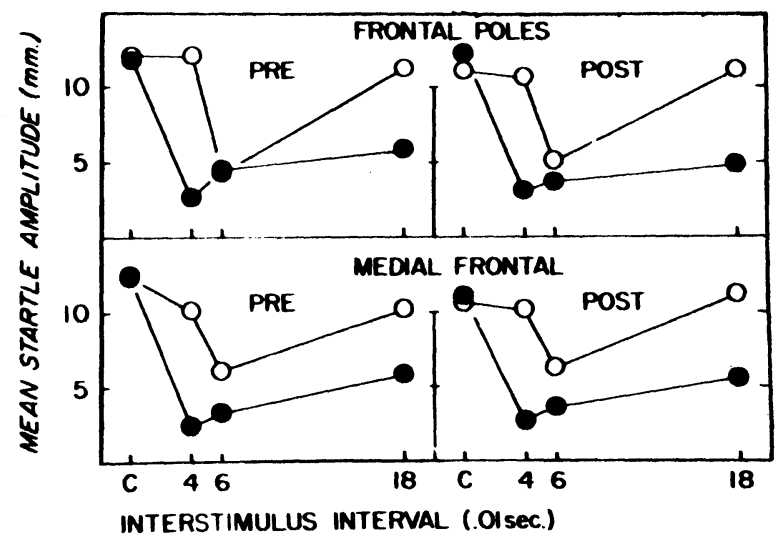

Fig. 4. Mean preoperative (PRE) and postoperative (POST) response amplitudes averaged over the animals in each surgical condition under control conditions (C) and under conditions where the startle pulse was preceded by the auditory prestimulus (filled circles) and the visual prestimulus (open circles) at the ISIs shown.

cortical areas, with occasional involvement of the olfactory bulbs. More posteriorly, the lesions extended to the genu of the corpus callosum, usually with some minor damage to the caudate-putamen.

Inspection of the brain damage with respect to postsurgical changes in control response amplitude indicated a correlation between amount of medial frontal cortex ablated bilaterally and postoperative increase in startle amplitude. An ordering of the six Ss with respect to these two variables yielded a Spearman rank-order correlation coefficient of $.60(\mathrm{p}<.10)$. Amount of damage to medial frontal cortex was assessed by placing an outline drawing of the section marked with a .01-in. grid of dots over each illustration and scoring the area of bilateral damage within the arbitrarily defined medial sector. The conformity of the rankings on brain damage and control response amplitude suggests that damage to the medial frontal cortex was critical in obtaining the postoperative elevation of response level. This anatomical correlation was examined more closely in Experiment II.

\section{EXPERIMENT II}

This experiment was run to provide more detailed information on the tentative finding, noted in Experiment I, that damage to medial frontal cortex was correlated with postoperative elevation of control response amplitude. For this purpose, the effects of ablation of either the frontal polar region or medial frontal cortex on both control and inhibited startle levels were examined.

\section{Behavioral Procedures}

A total of 14 animals were run under similar conditions to those used in Experiment I, with the exception that 64 trials were given in both the preoperative and postoperative test series. Each series consisted of 16 control trials, 8 trials where the startle pulse was preceded by an auditory prestimulus at each of three ISIs $(40,60$, and $180 \mathrm{msec})$, and 8 trials where the startle pulse was preceded by a visual prestimulus by the same three intervals. All stimulus parameters remained as in the previous experiment.

On the basis of the preoperative test, pairs of animals (weight range from 320 to $390 \mathrm{~g}$ at surgery) were matched according to control response amplitudes. Each pair member was then assigned to one of two conditions and underwent surgery aimed at ablating either the frontal polar region or the medial frontal cortex extending along the longitudinal fissure. General surgical procedures were the same for the two conditions and closely followed those used in Experiment I.

\section{Results and Discussion}

Behavioral. Figure 4 shows the results averaged over the seven animals within each surgical condition; group data are given for convenience, since all animals displayed highly similar behavioral functions. The obvious conformity of the pre- and postsurgical behavioral patterns for each group requires little comment; as in the first experiment, no changes in stimulus inhibition could be detected following surgical intervention.

Five of the rats tested showed a postoperative increase in control response amplitude, further supporting the implication of frontal cortical regions in behavioral inhibition. These five animals, however, were distributed approximately evenly across the two surgical conditions, three having sustained ablation of medial frontal cortex, while the remaining two underwent operations aimed at removing the frontal polar region.

Histological. Sample reconstructions on three frontal planes of ablations from the two surgical conditions are shown in Fig. 5. Substantial overlap between animals of the two groups with respect to brain damage on these planes was evident, with, typically, animals from the "frontal poles" group showing less than complete ablation of the medial region. Ablations in animals from the "medial frontal" group consistently extended more posteriorly, often involving the genu of the corpus callosum and the head of the caudate-putamen complex.

Generally, rather less brain damage was produced in the animals of Experiment II than in those of Experiment I as a consequence of the attempt to localize damage to separate frontal regions. It seems likely that this difference accounts for the smaller percentage of animals in Experiment II showing a postoperative enhancement of control response level.

Given the extensive overlap between the two groups in terms of frontal brain damage, the extent of bilateral medial frontal cortical damage on the three frontal planes shown in Fig. 5 was assessed for each $S$ in the same fashion as in Experiment I. Correlations of this ablation index with measures of postoperative startle responsivity showed a firm correlation between extent of medial cortical damage and response level on the initial four postoperative control trials as a percentage of response level on the same preoperative trials. This relationship is shown in the scatter diagram of Fig. 6, 
which yielded a product-moment correlation of .56 $(p<.05)$. Correlation of the index of medial cortical damage with the ratio of postoperative to preoperative response level taken over all control trials showed a similar, but less powerful relationship $(r=.31$, n.s. $)$. The diminution of the correlation with the response score taken over all control trials was chiefly contributed to by two animals with extensive medial frontal cortical damage, who showed an exaggerated level of response on the initial postoperative trials, which declined abruptly later in the test series. No reason for this within-series response decrement was apparent.

These results add some support to the implication of the medial frontal cortex of the rat in the inhibitory modulation of behavioral reactivity; in conjunction with this, the results are clear in showing that cortical damage which is followed by an augmentation of reactivity does not perturb the temporal pattern or degree of prestimulus inhibition.

\section{GENERAL DISCUSSION}

The expectation, based chiefly on findings of reflex modification in the cat, that lesions of frontal cortex
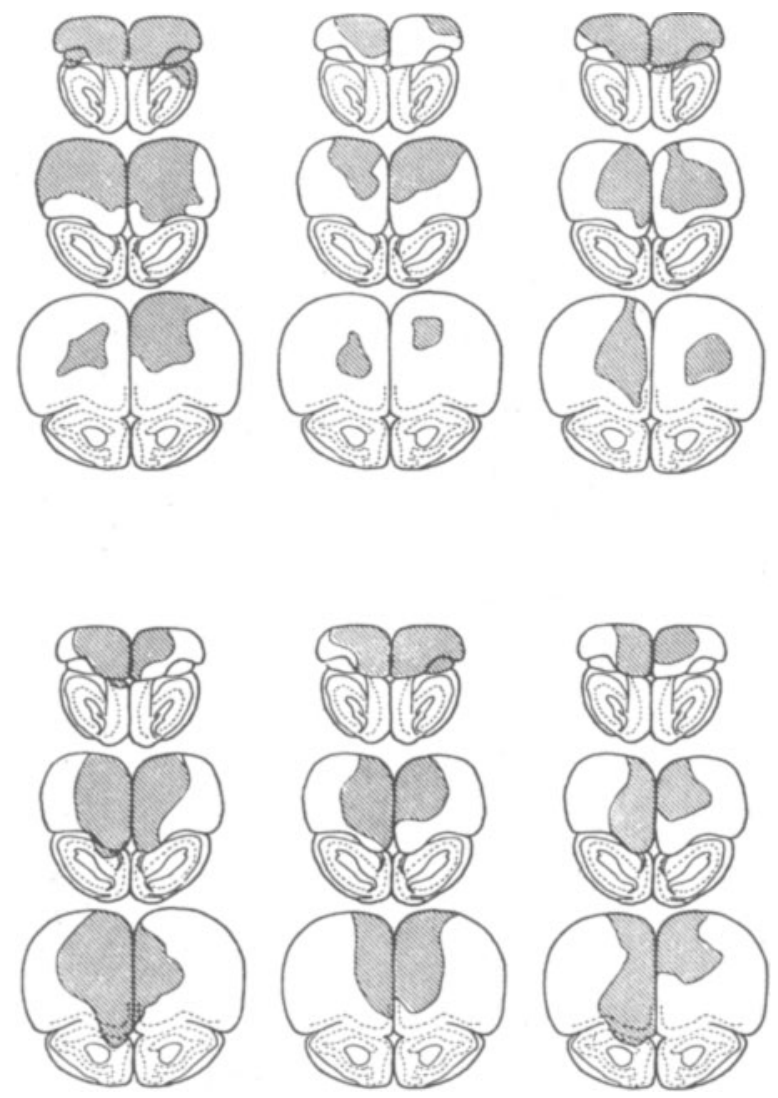

Fig. 5. Reconstructions of brain damage (hatched area) for three animals from the "frontal poles" group (top row) and three animals from the "medial frontal" group (bottom row) on illustrations of three frontal sections. after König and Klippel (1963).

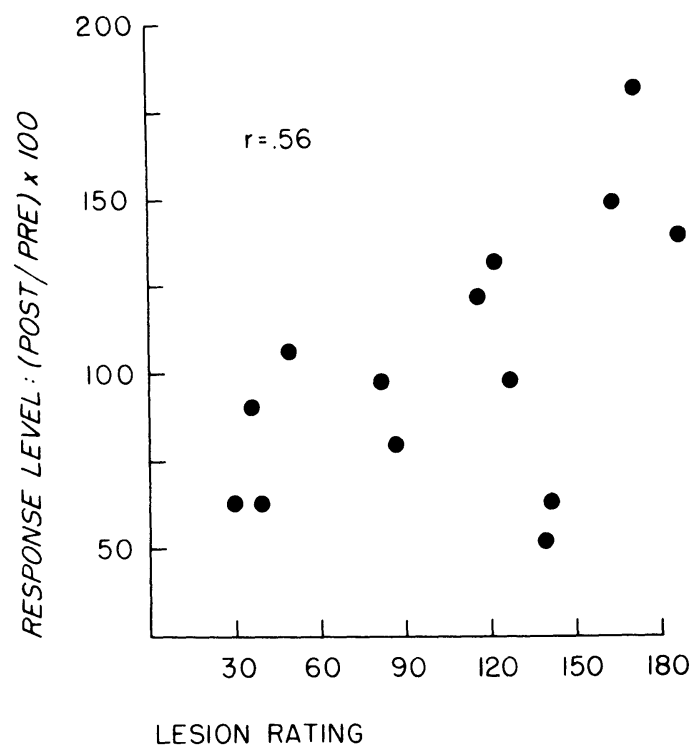

Fig. 6. Scatter diagram locating each animal with respect to extent of medial frontal cortical damage and percent postoperative change in response amplitude computed over the first four control trials.

would alter inhibition of the rat's acoustic startle reaction by visual and auditory prestimuli has not been substantiated. In conjunction with this, other work (Hammond, 1972) has shown that whole-brain transections (from posterior cortex caudally to just anterior to the preoptic area rostrally) designed to isolate the more caudally located startle-organizing system (Szabó \& Hazafi, 1965) from forebrain influences were similarly ineffective in altering prestimulus inhibition of the response. Together, these findings rule out any necessary contribution of frontal cortical regions to prestimulus inhibition.

The present finding that damage to medial frontal cortical areas appears to be critical in obtaining an apparent release from inhibition is in accord with the results reported by Divac (1971), who found a disturbance in the reversal of a learned discrimination (i.e., the animals continued to respond to a previously rewarded, but currently unrewarded, stimulus) following ablation of medial frontal cortex, but not following ablation of the frontal poles alone. This conformity of outcome, despite the diversity of the response index, suggests that the medial frontal cortex acts as part of a generalized behavioral inhibitory system, with nonspecific control over a range of behavioral systems. It is evident that this control does not extend to stimulus-produced inhibition of the startle reaction, for these functions remained intact following the otherwise effective lesion.

The present series of experiments, together with an earlier report dealing with the effects of lesions of the giant-celled pontine and medullary reticular nuclei (Hammond, 1973), was predicated on the assumption 
that inhibition of the rat's startle response is effected by a generalized behavioral inhibitory system which is anatomically differentiated and therefore susceptible to disruption by ablation. While no support for this general assumption has been provided by this work, other research (Hammond \& Ison, 1973) has shown that the inhibitory control of the startle response by a prior auditory stimulus is lost during various phases of barbiturate intoxication; these data provide some indication that the system which mediates stimulus inhibition is susceptible to degradation. Taken jointly, the failure to effect changes through localized brain lesions and the apparent success in effecting changes through drug administration suggests that there may be a diffuse inhibitory system which, while not anatomically differentiated from a reciprocally related excitatory system, is differentially sensitive to the action of barbiturates, and potentially other drugs. Should this be the case, placement of anatomically localized lesions would be of little analytic value.

\section{REFERENCES}

Brutkowski, S. Functions of prefrontal cortex in animals. Physiological Reviews, 1965, 45, 721-746.

Clemente, C. D. Forebrain mechanisms related to internal inhibition and sleep. Conditional Reflex, 1968, 3, 145-174.

Divac, I. Frontal lobe system and spatial reversal in the rat. Neuropsy chologia, 1971, 9, 175-183.

Griffin, J. P., \& Pearson, J. A. The effect of lesions of the frontal areas of the cerebral cortex on habituation of the flexor withdrawal response in the rat. Brain Research, 1968, 8, 177-184.

Hammond, G. R. Brain lesions and the acoustic startle reaction in rats. Unpublished doctoral dissertation, University of Rochester, 1972.

Hammond, G. R. Lesions of pontine and medullary reticular formation and prestimulus inhibition of the acoustic startle reaction in rats. Physiology \& Behavior, 1973, 10, 239-243.

Hammond, G. R., \& Ison, J. R. Stimulus-produced reflex inhibition during induction and recovery from barbiturate anesthesia. Journal of Comparative \& Physiological Psychology, 1973, 84, 436-444.

Hammond, G. R., McAdam, D. W., \& Ison, J. R. Effects of prestimulation on the electromyographic response associated with the acoustic startle reaction in rats. Physiology \& Behavior, 1972, 8, 535-537.

Hoffman, H. S., \& Searle, J. L. Acoustic variables in the modification of the startle reaction in the rat. Journal of Comparative \& Physiological Psychology, 1965, 60, 53-58.

Ison, J. R., \& Hammond, G. R. Modification of the startle reflex in the rat by changes in the auditory and visual environments. Journal of Comparative \& Physiological Psychology, 1971, 75, 435-452.

Ison, J. R., \& Krauter, E. E. Reflex inhibiting stimuli and the refractory period of the acoustic startle reflex in the rat. Journal of Comparative \& Physiological Psychology, 1973, in press.

König, J. F. R., \& Klippel, R. A. The rat brain. A stereotaxic atlas of the forebrain and lower parts of the brainstem. Baltim ore: Williams \& Wilkins, 1963.

Lynch, G. S. Separable forebrain sy stems controlling different manifestations of spontaneous activity. Journal of Comparative \& Physiological Psychology, 1970, 70, 48-59.

Roydes, R. L. Frontal lesions impair habituation of the head-shake response in rats. Physiology \& Behavior, 1970, 5 , 1133-1134.

Szabo, I., \& Hazafi, K. A. Elicitability of acoustic startle reaction with brain stem lesions. Acta Physiologica Academiae Scientiarum Hungericae, 1965, 27, 155-165.

Thompson, R. F., \& Spencer, W. A. Habituation: A model phenomenon for the study of neuronal substrates of behavior. Psychological Review, 1966, 173, 16-43.

\section{NOTE}

1. This control data has been reported in an earlier communication (Hamm ond, 1973).

(Received for publication November 15, 1973; revision received February 7,1974 .) 